\section{Soft X-ray resonant magnetic scattering from a Ni layer with modulated magnetic anisotropy}

\author{
A. Haznar, ${ }^{a, b}$ G. van der Laan, ${ }^{a *}$ S. P. Collins, ${ }^{a, c}$ \\ C. A. F. Vaz, ${ }^{\text {d J. A. C. Bland }}{ }^{d}$ and S. S. Dhesi $i^{c, e}$
}

${ }^{a}$ Magnetic Spectroscopy, Daresbury Laboratory, Warrington WA4 4AD, UK, ${ }^{b}$ Institute of Low Temperature and Structure Research, Polish Academy of Science, PO Box 1410, Wroclaw, Poland, ' Diamond Light Source Ltd, Rutherford Appleton Laboratory, Didcot, Oxon OX11 OQX, UK, ${ }^{d}$ Cavendish Laboratory, University of Cambridge, Madingley Road, Cambridge CB3 OHE, UK, and ${ }^{e}$ European Synchrotron Radiation Facility, BP 220, F-38043 Grenoble, France. E-mail:g.van_der_laan@dl.ac.uk

Soft X-ray resonant magnetic scattering studies on a magnetically modulated, but nominally chemically homogenous, $5 \mathrm{~nm}$ Ni layer in a $\mathrm{Cu} / \mathrm{Ni} / \mathrm{Cu} / \mathrm{Co} / \mathrm{NiO} / \mathrm{GaAs}(110)$ system are reported. It was possible to estimate the main chemical structure of the sample on the basis of the results from specular reflectivity and rocking scans, probing the scattering vector components in the perpendicular and in-plane direction to the Ni wires, respectively. The magnetic scattering using polarized X-rays demonstrates the magnetic modulation of the $\mathrm{Ni}$ layer.

Keywords: X-ray resonant magnetic scattering; nanostructures.

\section{Introduction}

In the pursuit of ever-increasing ultrahigh-density storage capabilities, the size dependence of the spin configurations and reversal processes of magnetic nanostructures produced by lithographic patterning of thin magnetic films are of significant scientific and technological interest. While isolated 'island' magnetic structures, such as dots, wires and other special geometries, have already been studied (Dürr et al., 1999; Dudzik et al., 2000; Jaouen et al., 2002; Chesnel et al., 2002), the experimental realisation of patterned magnetic structures in a nominally chemically homogeneous film remains an important goal. Controlling both the intrinsic properties of the material and the sample dimensions of the magnetic domains in continuous films can open up new avenues for fundamental studies and device applications. Such structures can be used as a planar patterned magnetic medium, without breaking the homogeneity of the magnetic film which avoids reduced Curie temperature effects (Cowburn et al., 1997). It also introduces a new way of controlling spin configurations instead of stabilizing the natural domains (KrusinElbaum et al., 2001). Locally controlled spin configurations do not have lateral dimension limitations and thus provide a reproducible switching mechanism, unlike geometrically restricted domains (Taniyama et al., 1999; Ebels et al., 2000).

Recently, Li et al. (2002) and Bland et al. (2002) demonstrated a novel approach to controlling the magnetic structure in chemically homogenous films via the artificial modification of substrate surface properties prior to growth. Selective epitaxial growth introduces an alteration between single-crystal and polycrystalline structures in the film, according to the substrate patterning. The modulated singlecrystal/polycrystalline substrate surface was used to locally modify the magnetic anisotropy in the subsequently deposited magnetic $\mathrm{Ni}$ film, which induces the desired magnetic structure. The well studied $\mathrm{Cu} / \mathrm{Ni} / \mathrm{Cu}(001)$ system was used to obtain domains with perpendicular magnetic anisotropy (PMA), which is attributed to the magnetoelastic interaction induced by the $\mathrm{Ni} / \mathrm{Cu}(001)$ single-crystal interface (O'Brien \& Tonner, 1994), while in the polycrystalline Ni domains the magnetization lies in the film plane owing to the dominant demagnetizing field.

$\mathrm{X}$-ray reflectivity provides a routine method of gathering structural information, such as layer thickness, effective electron density and interfacial roughness (Daillant \& Gibaud, 1999; Als-Nielsen \& McMorrow, 2000). However, the capabilities of conventional X-ray reflectivity are limited when it is necessary to distinguish between the structural parameters from layers of neighbouring elements in the periodic table, such as $\mathrm{Co}, \mathrm{Ni}$ and $\mathrm{Cu}$, as in the case of our system (see Fig. 1). A way of enhancing the chemical contrast is to take advantage of the tunability of the incident photon energy, which has become possible with the advent of synchrotron radiation (Kao et al., 1994; Tonnerre et al., 1998; Sève et al., 1999). The scattering factor will be enhanced near the absorption edges of the various elements in the sample. This resonant enhancement can be used to tune into the element-specific scattering cross section and to increase the sensitivity to the structural features. For $3 d$ transition metals we use the excitation of $2 p$ electrons into unoccupied $3 d$ states resulting in strong absorption edges with energies in the soft X-ray region (van der Laan \& Thole, 1991).

In this paper we will present soft $\mathrm{X}$-ray resonant magnetic scattering (SXRMS) results on the $\mathrm{Cu} / \mathrm{Ni} / \mathrm{Cu}$ system in which the Ni layer has a wire pattern of modulated magnetic anisotropy (Fig. 1). Since the properties of the sample are closely related to the structure, we demonstrate that SXRMS is a chemical-structure- and magneticstructure-sensitive and element-specific technique, which shows a large potential to study such complex magnetic systems. Very few SXRMS studies have been reported so far on artificially patterned magnetic samples (Chesnel et al., 2001, 2002) and it is primarily the purpose of the current paper to demonstrate the potential of the technique, rather than to indulge in a detailed theoretical analysis of the scattering results.

\section{Sample preparation}

Using $\mathrm{GaAs}(001)$ as a substrate, a pattern was prepared of a wire array of width $2 \mu \mathrm{m}$ and separation $4 \mu \mathrm{m}$, as described by Li et al.

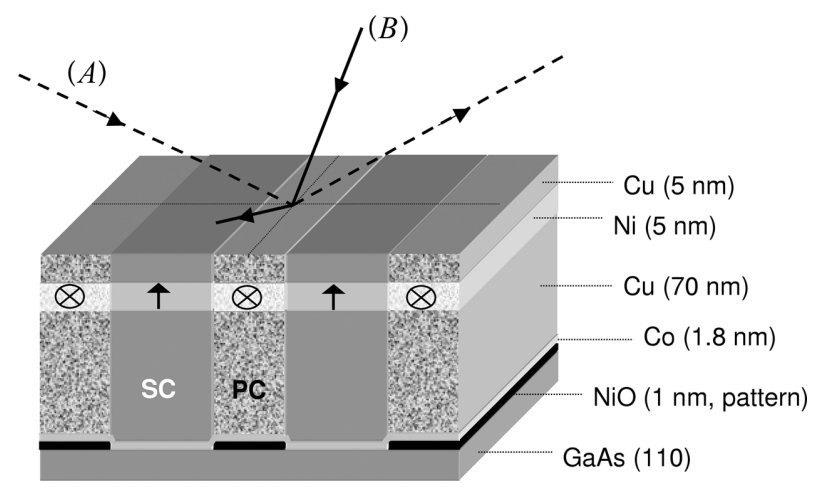

Figure 1

Schematic of the measured sample $\mathrm{Cu}(5 \mathrm{~nm}) / \mathrm{Ni}(5 \mathrm{~nm}) / \mathrm{Cu}(70 \mathrm{~nm}) /$ Co $(1.8 \mathrm{~nm}) / \mathrm{NiO}(1 \mathrm{~nm}) / \mathrm{GaAs}(110)$. The thickness of the layers is given according to the characterization by Li et al. (2002). Polycrystalline and singlecrystalline regions of the sample are indicated by PC and SC, respectively. Also shown are the experimental scattering geometries for $(A)$ X-rays perpendicular to the wires and $(B) \mathrm{X}$-rays parallel to the wires. 
(2002) and Bland et al. (2002). A schematic is shown in Fig. 1. The pattern was obtained by optical lithography and a subsequent lift-off of $1 \mathrm{~nm}$-thick Ni. The ultrathin pattern was then oxidized in air to become an ultrathin $\mathrm{NiO}$ pattern. The patterned substrate was annealed at $773 \mathrm{~K}$ for $2 \mathrm{~h}$ before film growth at room temperature. Continuous films of $\mathrm{Cu}(5 \mathrm{~nm}) / \mathrm{Ni}(5 \mathrm{~nm}) / \mathrm{Cu}(70 \mathrm{~nm}) / \mathrm{Co}(1.8 \mathrm{~nm})$ were deposited onto the substrate in UHV using molecular beam epitaxy. The $1.8 \mathrm{~nm}$-thick Co is used as a seed layer to promote epitaxial growth on the GaAs substrate. The thickness of $5 \mathrm{~nm}$ for the Ni layer is chosen in order to obtain a strong PMA (O'Brien \& Tonner, 1994), and the $5 \mathrm{~nm}$-thick $\mathrm{Cu}$ overlayer is used to prevent oxidation. While the films grown on the ultrathin $\mathrm{NiO}$ patterns are polycrystalline, those grown directly onto the GaAs surface are single-crystal films. The results have been confirmed by in situ reflection high-energy electron diffraction (RHEED) analysis. Magneto-optical Kerr effect (MOKE) measurements were performed to obtain the hysteresis loops. While magnetic force microscopy (MFM) was used to study the magnetic domain structures, atomic force microscopy (AFM) was used to examine the sample surface (Li et al., 2002; Bland et al., 2002). Using these combined methods one can characterize the magnetic structure of the sample, and using the RHEED technique one can confirm the coexistence of polycrystalline and single-crystal regions in the Ni layer. However, in order to reveal the internal structure of the system, a depth-sensitive probe, such as SXRMS, is required.

\section{X-ray magnetic scattering}

The SXRMS measurements were performed on beamline ID08 at the European Synchrotron Radiation Facility (ESRF) in Grenoble using the six-circle ultrahigh-vacuum diffractometer (Dhesi et al., 2004). Synchrotron radiation with linear and circular polarization in the energy region between 700 and $950 \mathrm{eV}$ was employed at grazingincidence angles $\theta$ between 0.1 and $22.5^{\circ}$. The scattered X-rays were detected using a photodiode (mounted behind a rectangular aperture) in vertical geometry. For the magnetic characterization the sample magnetization was applied parallel to the wires using an inplane magnetization coil providing a field up to $50 \mathrm{mT}$. Since the coercive field of the sample is $20 \mathrm{mT}$, this is sufficient to saturate the sample.

Two different types of scans were employed, namely rocking scans and reflectivity scans, which in reciprocal space measure the scattering signal along $q_{x}$ and $q_{z}$, respectively (see Fig. 2). Here, $q_{z}$ is the component perpendicular to the surface, while $q_{x}$ is the component of the scattering vector in-plane with the sample surface and perpendicular to the wires. The real-space geometries $A$ and $B$ used for rocking scans and reflectivity scans, respectively, are schematically illustrated in Fig. 1, and below we will describe these scans to improve the understanding of the experimental results given in this paper.

\section{1. $q_{x}$ scan with fixed $2 \theta$ (rocking scan)}

The scattering is measured as a function of $q_{x}$ using a transverse scan with the scattering plane perpendicular to the wires (geometry $A$ in Fig. 1) by rocking the sample at a fixed detector position. This rocking scan provides information about the in-plane periodicity of the charge and/or magnetic configuration. The scan can be perceived as a so-called grating scan with fixed $2 \theta$.

For ingoing grazing angle $(\theta+\alpha)$ and outgoing grazing angle $(\theta-\alpha)$ we obtain the components of the scattering vector as

$$
\begin{aligned}
& q_{x}=(4 \pi / \lambda) \sin \theta \sin \alpha, \\
& q_{z}=(4 \pi / \lambda) \sin \theta \cos \alpha,
\end{aligned}
$$

where $\lambda$ is the wavelength of the incident radiation and $\alpha$ is the rocking angle, i.e. the angle between the scattering vector $\mathbf{q}$ and the surface normal. The wavevector transfer $\mathbf{q}=\mathbf{k}-\mathbf{k}^{\prime}$ is the difference between the wavevectors $\mathbf{k}$ and $\mathbf{k}^{\prime}$ of the ingoing and outgoing X-rays, respectively. Thus the rocking scan describes a circle in reciprocal space given by

$$
\begin{gathered}
|\mathbf{q}|=\left(q_{x}^{2}+q_{z}^{2}\right)^{1 / 2}=(4 \pi / \lambda) \sin \theta, \\
q_{z} / q_{x}=\cot \alpha .
\end{gathered}
$$

In our case the incident angle and rocking angle are small, so that equations (1) and (2) simplify to $q_{x} \simeq 4 \pi \theta \alpha / \lambda$ and $q_{z} \simeq 4 \pi \theta / \lambda$. Thus, with fixed $2 \theta$, the rocking scan measures the scattering intensity along the $q_{x}$ direction at constant $q_{z}$.

Fig. 2 illustrates the reciprocal space map showing the specular rod and the equidistantly spaced diffraction rods, corresponding to our patterned sample. The horizontal lines at $q_{z}=0.03$ and $0.09 \AA^{-1}$ represent the $q_{x}$ scans with $2 \theta=4^{\circ}$ and $12^{\circ}$, respectively, for a wavelength $\lambda=14.5 \AA$ corresponding to the $\mathrm{Ni} L_{3}$ resonance. Access in reciprocal space is restricted by the horizon condition, $|\alpha| \leq \theta$, to the region on the map satisfying

$$
q_{z}^{2} \geq(4 \pi / \lambda)\left|q_{x}\right|-q_{x}^{2}
$$

\section{2. $q_{z}$ scan (specular scan)}

The reflectivity is measured as a function of $q_{z}$ using a $\theta-2 \theta$ scan with the scattering plane parallel to the wires (geometry $B$ in Fig. 1). Specular reflection results in $q_{x}=0$ and $q_{z}=(4 \pi / \lambda) \sin \theta$. The specular scan, where $q_{z}$ is varied by scanning $\theta$ and $2 \theta$, provides information about the structural and magnetic depth profile of the sample. In the reciprocal space map of Fig. 2, the $q_{z}$ scan is shown by the vertical line $\left(q_{x}=0\right)$.

\section{Resonant enhancement}

The huge enhancement in the scattering intensity across the $\mathrm{Ni}$ resonance is illustrated in Fig. 3, which shows the energy dependence

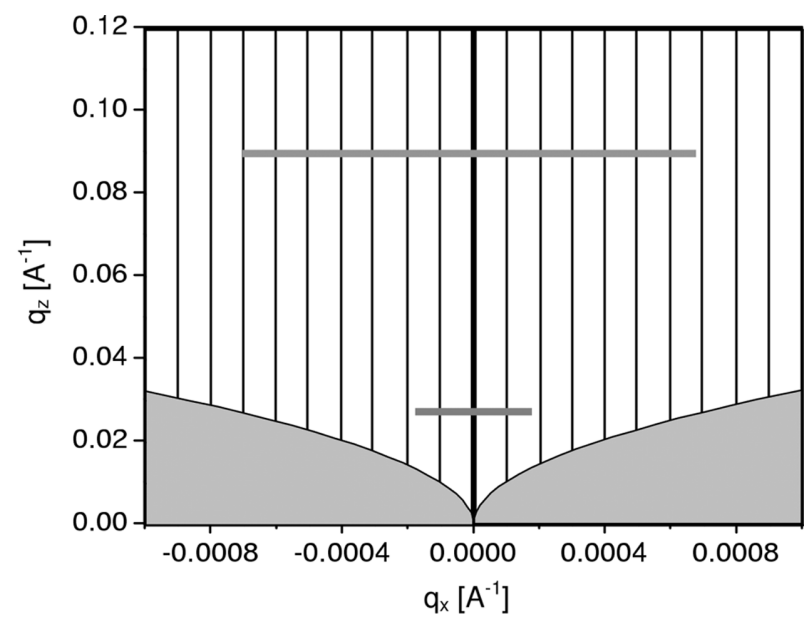

Figure 2

The reciprocal plane $\left(q_{x}, q_{z}\right)$ showing the specular rod $\left(q_{x}=0\right)$ and the equidistantly spaced diffraction rods $\left(\Delta q_{x}=0.0001 \AA^{-1}\right.$ for our sample). The $q_{x}$ scans (rocking scans) for $2 \theta=4^{\circ}$ and $12^{\circ}$ at the $\mathrm{Ni} L_{3}$ resonance $(\lambda=14.5 \AA)$ are shown by the thick grey horizontal lines. The $q_{z}$ scan (specular scan) is along the specular $\operatorname{rod}\left(q_{x}=0\right)$ as shown by the thick black vertical line. The grey areas indicate the inaccessible region. 
of the scattering intensity measured using a $q_{x}$ scan with $2 \theta=10^{\circ}$. A strong intensity increase is observed at the energies of the $\mathrm{Ni} L_{3}$ and $L_{2}$ edges, located at 852 and $870 \mathrm{eV}$, respectively. The presence of many higher-order diffraction peaks in the rocking scan shows that the patterned sample diffracts as a grating.

Using polarized X-rays the resonant scattering becomes sensitive to the magnetic order of the sample (van der Laan, 1999). By tuning the energy to the core resonance the weak magnetic signal can be increased by orders of magnitude when the magnetic orbitals are directly involved in the electric dipole transition, such as the $2 p \rightarrow 3 d$ excitation in first-row transition metals. The energy-dependent scattering amplitude $f_{i}$ for coherence resonant magnetic scattering can be written, to first order in the magnetization, as

$$
f_{i}(\omega)=\left(\boldsymbol{\varepsilon}^{\prime *} \cdot \boldsymbol{\varepsilon}\right) F^{0}-i\left(\boldsymbol{\varepsilon}^{\prime *} \times \boldsymbol{\varepsilon}\right) \cdot \hat{\mathbf{M}}_{i} F^{1},
$$

where $\varepsilon\left(\varepsilon^{\prime}\right)$ is the unit polarization vector of the incident (scattered) $\mathrm{X}$-ray beam, $\hat{\mathbf{M}}_{i}$ is the magnetization unit vector of the $i$-th site, and $F^{0}$ and $F^{1}$ are the charge and magnetic part of the resonance amplitude (Hannon et al., 1988). The first term in equation (6) has the same appearance as the non-resonant Thomson scattering, while the second term is unique to the resonance. The polarization dependence, given by the vector products, is of great benefit to determine the alignment of the magnetic moments. With $\sigma$-polarized light (i.e. linear polarization perpendicular to the scattering plane) one probes the charge due to $\sigma \rightarrow \sigma^{\prime}$ scattering and also the magnetization component (along $\mathbf{k}^{\prime}$ ) in the scattering plane due to $\sigma \rightarrow \pi^{\prime}$ scattering (van der Laan et al., 2000). With circularly polarized light one probes magnetic components both within and perpendicular to the scattering plane. If the difference signal between left- and right-circularly polarized light is taken to examine the magnetization, then only the component within the scattering plane and perpendicular to $q_{z}$ contributes to the magnetic signal. Also, helical and chiral magnetic structures, although not considered in this paper, can give magnetic scattering peaks (Dürr et al., 1999).

The scattering intensity contains the modulus square of the amplitude $f_{i}(\omega)$. When the charge and magnetic periodicity is the same, there will be an overlap of the magnetic and charge peaks. Then, in order to study the magnetism one can sometimes exploit the interference between the two amplitudes which results in a change in the scattering signal upon reversal of the sample magnetization.

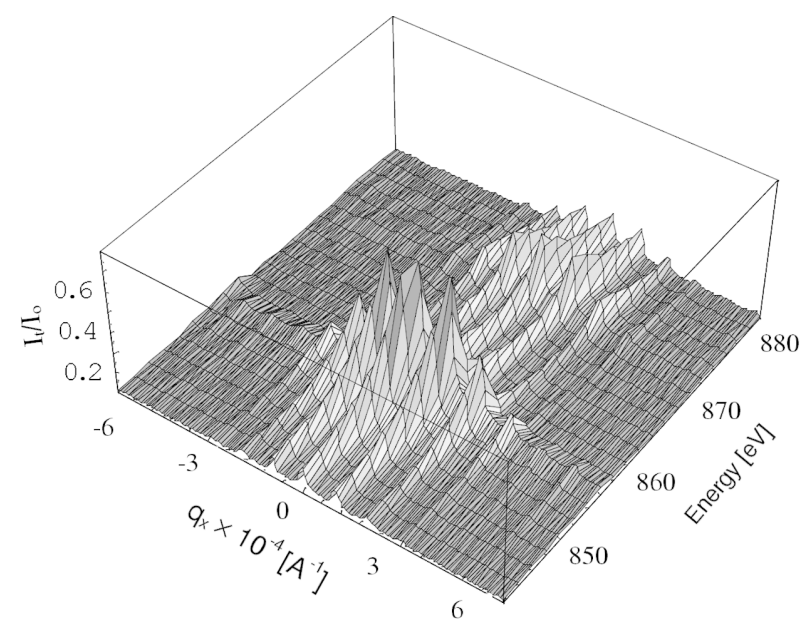

Figure 3

Two-dimensional plot of the photon-energy- and $q_{x}$-dependence of the scattered intensity measured by rocking scans with $2 \theta=10^{\circ}$ across the Ni $L_{2,3}$ resonance.
Dichroic intensity changes of this type, upon reversal of either the circular polarization or magnetic vectors, scale with $\left(\mathbf{k}+\mathbf{k}^{\prime} \cos 2 \theta\right) \cdot \hat{\mathbf{M}}_{i}$ (Lovesey \& Collins, 1996).

\section{Results and discussion}

\subsection{Structural profile of the sample}

5.1.1. Element-specific depth structure. Although our main interest is the magnetic properties of the system, the chemical structure needs to be characterized first in order to evaluate the magnetic contribution. Fig. 4 shows $q_{z}$ scans measured at three different photon energies. They are remarkably different to each other. The soft X-ray wavelengths are too long $(\sim 15 \AA)$ to reveal Bragg scattering from atomic planes and, apart from their interfaces, the layers are expected to be homogeneous in depth. However, the scattered intensity still depends on the X-ray penetration depth, which is material dependent. It strongly varies with scattering angle and photon energy, especially near the critical angle and across the resonances. Intensity oscillations, known as Kiessig fringes, arise from the interference between the scattering amplitudes from the different layer interfaces. The periods of the Kiessig oscillations are inversely proportional to the layer thicknesses.

At $700 \mathrm{eV}$, the photon energy is well below the $\mathrm{Co}, \mathrm{Ni}$ and $\mathrm{Cu} L_{2,3}$ absorption edges, which means that the penetration depth is large enough for the X-rays to reach the substrate. The short-period oscillation observed on the $q_{z}$ scan at $700 \mathrm{eV}$ (Fig. 4) corresponds to a thickness of the $\mathrm{Cu}$ underlayer of $530 \AA$, which is smaller than the value of $700 \AA$ estimated from the preparation method. By tuning the energy to the $\mathrm{Ni} L_{3}$ edges at $852.7 \mathrm{eV}$, the scattered intensity becomes sensitive to the Ni layer, and the long-period oscillation in Fig. 4 corresponds to a thickness of $70 \AA$. Owing to the increased absorption at the $\mathrm{Ni}$ edge the short-period oscillation related to the thick $\mathrm{Cu}$ underlayer is strongly suppressed. The thickness of the $\mathrm{Cu}$ capping layer was obtained from a measurement at $900 \mathrm{eV}$, which is just below the $\mathrm{Cu} L_{3}$ edge. Fig. 4 shows a short-period oscillation related to the thick $\mathrm{Cu}$ underlayer as well as an oscillation that is about ten times longer and corresponds to the $\mathrm{Cu}$ capping layer of $50 \AA$.

The patterned sample gives an additional feature in the reflectivity around $q_{z}=0.05 \AA^{-1}$ (Fig. 4). Such a feature is normally not observed for a multilayer which is in-plane homogeneous. Therefore, we tentatively ascribe this strong dip at small $q_{z}$ value to the in-plane

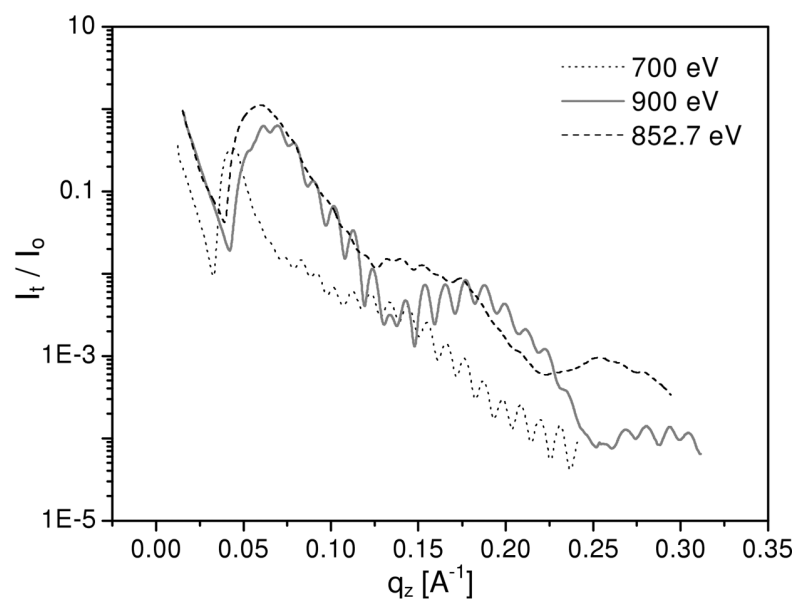

Figure 4

Specular rod scans $\left(q_{z}\right.$ scans) at photon energies of $700 \mathrm{eV}$ (below all relevant edges), $852.7 \mathrm{eV}$ (at the $\mathrm{Ni} L_{3}$ edge) and $900 \mathrm{eV}$ (just below the $\mathrm{Cu} L_{3}$ edge). The periods of the Kiessig fringes correspond to the layer thicknesses in reciprocal space. 
periodicity in the system. The $\mathrm{NiO}$ thin film pattern, the polycrystalline and single-crystal regions, the different oxidation for the top layers in the polycrystalline and single-crystalline regions will all act together as a diffraction grating. Scattered amplitudes from the different regions of the sample interfere owing to phase differences and might give rise to an additional variation in the scattered intensity.

5.1.2. In-plane structural profile. As already demonstrated by Fig. 3, the most direct way to extract information concerning the inplane structure is by performing rocking scans. Figs. 5 and 6 show the $q_{x}$ scans measured with $2 \theta=4^{\circ}$ and $12^{\circ}$, respectively, at different photon energies. The separation of $\Delta q_{x}=0.0001 \AA^{-1}$ between the diffraction peaks, which are located around the specular reflection $\left(q_{x}=0\right)$, corresponds to a wire periodicity that matches precisely the period of $6 \mu \mathrm{m}$, laid down by lithography.

Fig. 5 shows the $q_{x}$ scans at $2 \theta=4^{\circ}$, which is near the critical angle of total reflection where the sampling depth is minimum. In this case the scattering will be strongly surface sensitive so that only the capping layer will contribute. Since AFM measurements did not reveal any correlated roughness on the surface (Li et al., 2002), the appearance of first-order diffraction peaks suggest a different oxidation for the single-crystalline and polycrystalline regions of the $\mathrm{Cu}$ capping layer. It is also clear from Fig. 5 that the normalized intensity profile near the total-reflection angle is almost independent of the photon energy.

At increasing Bragg angles the penetration depth becomes larger, so that the scattering signal will gradually give more information about the buried layers. Fig. 6 shows the $q_{x}$ scans with $2 \theta=12^{\circ}$. As with the $q_{z}$ scans in Fig. 4, switching the photon energy changes the scattering signal depending on the scattering factors of the elements involved. The relative peak intensities as well as the peak shape depend on the specific photon energy and Bragg angle. The envelope function of the peak intensities gives the form factor, which can provide information about the average structure of the wires. When the photon energy is tuned to the $\mathrm{Ni} L_{3}$ or $\mathrm{Cu} L_{3}$ resonance, extra structure is observed in the specular peak, while also an additional shoulder appears on the left-hand side of each diffraction peak. We tentatively ascribe this to the interference between the scattered amplitudes of the grating profile at different depths in the sample. The peak shape of each diffraction peak is quite similar, and therefore can be considered as a structure factor that provides information about the periodicity of the pattern summed over the effective

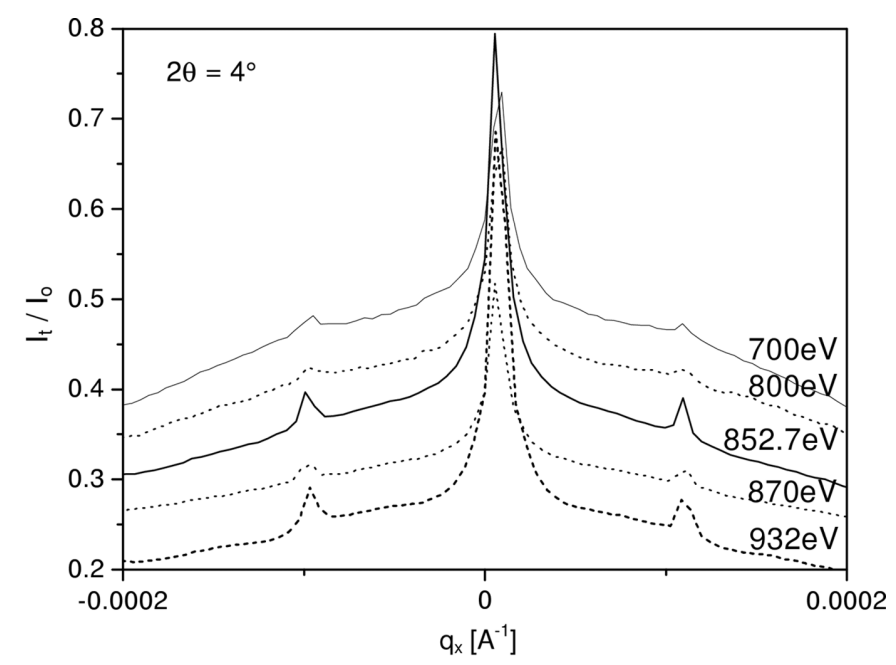

Figure 5

The $q_{x}$ scans at $2 \theta=4^{\circ}$ measured at different photon energies. sampling depth. However, without any detailed calculations it is very difficult to assign the peak shape to particular structural features of the sample.

\subsection{Magnetic modulation of the sample}

The $\mathrm{Ni}$ layer is of main interest for the $\mathrm{Cu} / \mathrm{Ni} / \mathrm{Cu}$ magnetically modulated system. In order to be selective to the $\mathrm{Ni}$ layer and to obtain an optimal magnetic scattering cross section, the photon energy was tuned to the $\mathrm{Ni} L_{2,3}$ absorption edge. We will first demonstrate the presence of both in-plane and perpendicular magnetic domains in the Ni layer by using the magnetic direction selectivity of SXRMS. Thereafter, we report on the magnetic depth profile using the scattering angle and photon energy dependence of the magnetic circular dichroism (MCD) asymmetry ratio in specular reflectivity.

5.2.1. Coexistence of in-plane and perpendicular magnetic domains. The energy dependence of the specular reflectivity was measured across the $\mathrm{Ni}$ edges at $2 \theta=8^{\circ}$ (i.e. $q_{x}=0, q_{z}=0.06 \AA^{-1}$ ) using $\sigma$-polarized light in geometry $A$. According to the vector product in equation (6), $\sigma$-polarized light can detect the magnetization component in the scattering plane along $\mathbf{k}^{\prime}$. Since the scattering plane is perpendicular to the wires in geometry $A$ (c.f. Fig. 1 ), the magnetic scattering vanishes for the in-plane (parallel-to-wire) domains, and only the magnetic component perpendicular to the surface contributes to the magnetic scattering.

Fig. 7 shows the difference in scattered intensity from two different magnetic configurations of the sample. In the first configuration the sample is in remanence, where we expect it to contain both in-plane and perpendicular magnetic domains. In the second configuration all magnetic domains have been aligned in-plane along the wire direction using the magnetization coil. The difference between both configurations gives the magnetic signal from the domains with perpendicular magnetization. The non-zero difference signal shown in Fig. 7 proves the presence of domains with perpendicular magnetization. Unfortunately, we were not able to observe the much weaker first-order magnetic scattering, since the structural and magnetic periods overlap, resulting in charge and magnetic scattering peaks at the same position in reciprocal space. We also studied the magnetic behaviour of the sample using circular polarization in geometry $B$ with the light helicity vector aligned either parallel or antiparallel to the magnetization of the wires, resulting in scattering

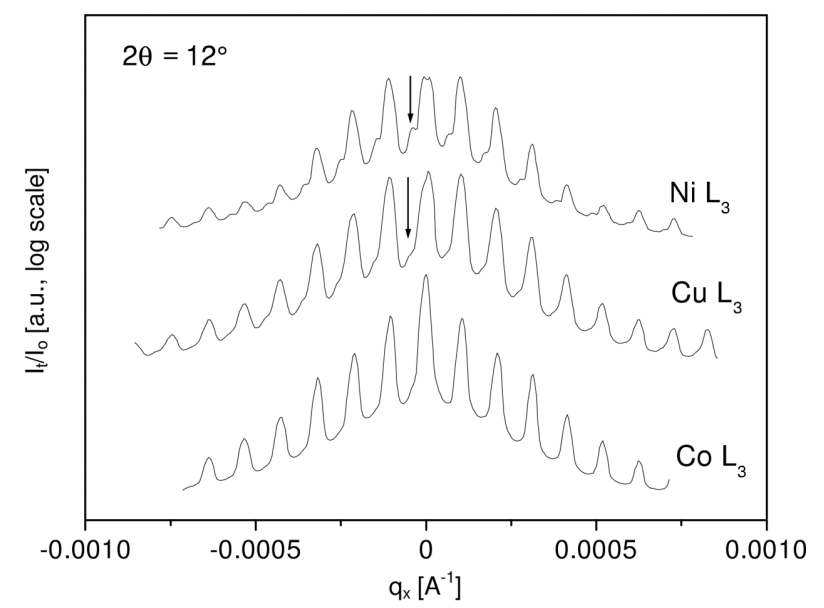

Figure 6

The $q_{x}$ scans at $2 \theta=12^{\circ}$ measured at the photon energies of the $\mathrm{Ni}, \mathrm{Cu}$ and $\mathrm{Co}$ $L_{3}$ resonances. The curves have been offset for clarity. The arrows point at the shoulder visible at the left-hand side of the specular peak. 
signals denoted as $I^{+}$and $I^{-}$, respectively. With circular polarization we observe differences between the scattering signals from the sample in remanence and with only in-plane magnetically aligned domains. Fig. 8 shows the asymmetry ratio $\left(I^{+}-I^{-}\right) /\left(I^{+}+I^{-}\right)$for both types of magnetic configuration at $2 \theta=10^{\circ}$ (i.e. $q_{x}=0, q_{z}=$ $0.076 \AA^{-1}$ ). For the remanent sample the helicity vector of the circularly polarized light was reversed, while for the in-plane magnetically aligned domains the magnetization was reversed using the in situ magnetization coil. With circular polarization, both inplane and perpendicular domains give a non-zero magnetic scattering. If the perpendicularly aligned magnetic domains remain unchanged, the contribution to the MCD in reflectivity will arise only from the reversal of the in-plane magnetic moments. For that reason the signal will be approximately twice as large for measurements with

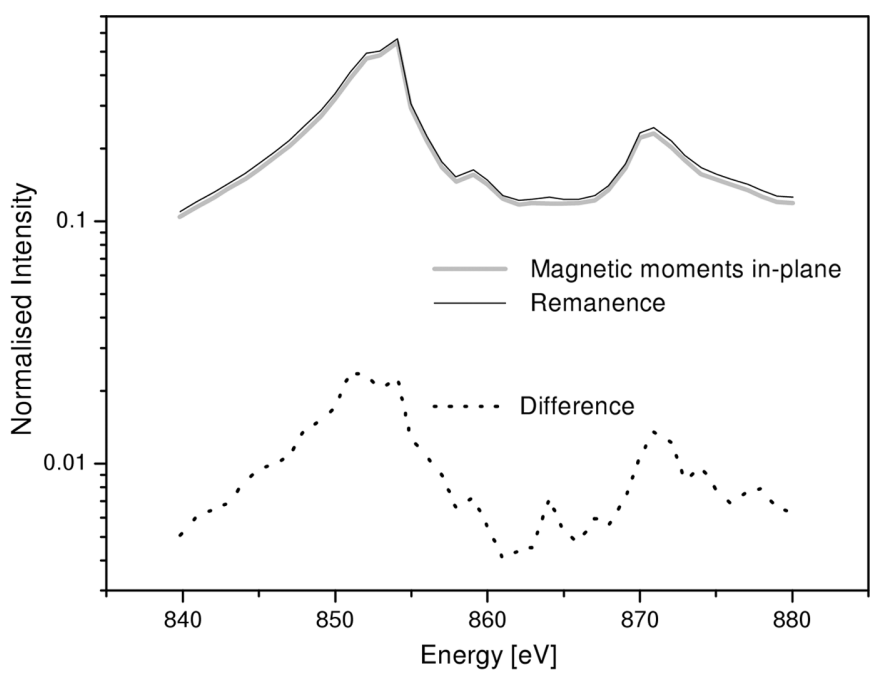

\section{Figure 7}

Specular reflectivity of the magnetic configuration in remanence (with coexisting in-plane and perpendicular magnetic domains) (black line) and the magnetic configuration with all magnetic moments aligned in-plane (thick grey line) together with the resulting difference spectrum (dashed line). The specular reflectivity was measured as a function of photon energy at $2 \theta=8^{\circ}$ using $\sigma$-polarized light.

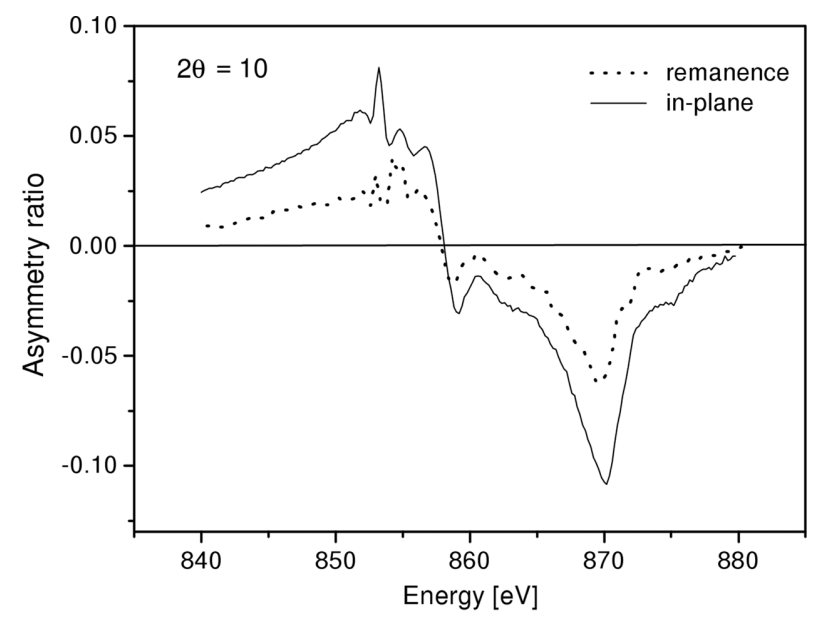

Figure 8

Energy dependence of the asymmetry ratio $\left(I^{+}-I^{-}\right) /\left(I^{+}+I^{-}\right)$in specular reflection at $2 \theta=10^{\circ}$ for the sample in remanence (dotted line) and with inplane applied magnetic field (drawn line). The signal $I^{+}\left(I^{-}\right)$is defined as the specular reflectivity with the sample magnetization aligned parallel (antiparallel) to the X-ray helicity vector. in-plane magnetic field than under remanence conditions, where inplane and perpendicular magnetic moments coexist.

5.2.2. Magnetic depth profile. The magnetic depth profile was studied using the scattering-angle- and photon-energy-dependence of the MCD asymmetry ratio in specular reflectivity (in geometry $B$ ). All results presented below are for the $I^{+}$and $I^{-}$signals from the magnetic configuration in which all magnetic moments are aligned inplane and are reversed using the magnetization coil.

Fig. 9 shows the dependence of the asymmetry ratio $\left(I^{+}-I^{-}\right) /\left(I^{+}+I^{-}\right)$on the Bragg angle measured at the energies of the $\mathrm{Ni} L_{2}$ and $L_{3}$ edges. The insert shows the difference signal $\left(I^{+}-I^{-}\right)$, i.e. the MCD, shifted by +0.08 . Because this difference signal varies strongly with Bragg angle and can also change sign, it is advantageous to present the scattering signal in the form of an asymmetry ratio. However, the short-period oscillations shown in the asymmetry ratio can also be produced by minima in the charge reflectivity. No magnetic signal is observed at $2 \theta \leq 5^{\circ}$, where the $\mathrm{X}$-rays only penetrate the $\mathrm{Cu}$ capping layer. This proves that the capping layer is not magnetic. Strong changes in the reflectivity are observed at higher angles, clearly demonstrating the large sensitivity of the asymmetry ratio to the magnetic structure and magnetic interfaces.

We have already presented in Fig. 8 (drawn line) the asymmetry ratio for in-plane magnetically aligned domains over the $\mathrm{Ni} L_{2,3}$ resonance in geometry $B$ with $2 \theta=10^{\circ}$. In Fig. 10 we present a series of such energy scans for different Bragg angles at grazing incidence. Remarkably strong spectral changes can be observed as a function of the Bragg angle.

The thick line in Fig. 10 gives the sum of the reflectivity signals $\left(I^{+}+I^{-}\right)$. The observed evolution of the sum reflectivity for different Bragg angles resembles that for the grazing-incidence reflectivity spectra of the thin $\mathrm{NiO}$ film (Alders et al., 1997; van der Laan, 1999). This NiO film was measured with $\sigma$-polarization and the reflectivity results gave good agreement with an optical model calculation. Of course, our layer contains $\mathrm{Ni}$ metal, rather than $\mathrm{NiO}$, which has a somewhat different $\mathrm{Ni} L_{2,3}$ multiplet structure. However, both

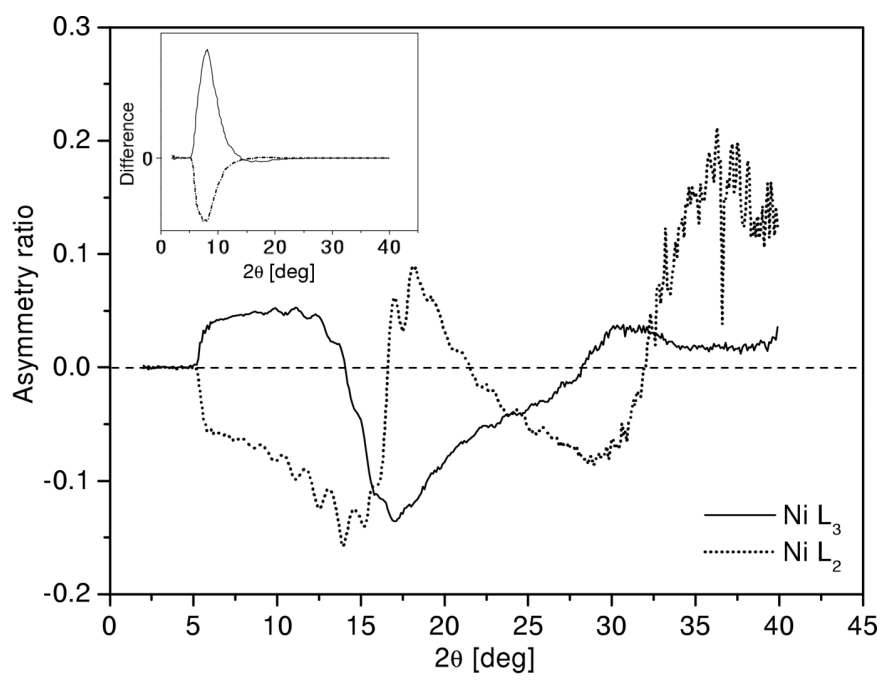

Figure 9

The $2 \theta$ dependence of the asymmetry ratio of the specular reflectivity at the photon energy of the $\mathrm{Ni} L_{3}$ (drawn line) and $\mathrm{Ni} L_{2}$ (dotted line) resonance. Note the strong reduction in amplitude of the Kiessig fringes for the $\mathrm{Ni} L_{3}$ resonance owing to the shorter absorption length at this stronger absorption edge. Insert: difference between the scattering signal with parallel and antiparallel applied magnetic field for the $\mathrm{Ni} L_{3}$ (drawn line) and $\mathrm{Ni} L_{2}$ (dashed line) resonance. 
systems show quite similar variations as a function of $\theta$; in particular there is a peak, consisting of several components, at both the $L_{3}$ and $L_{2}$ edge around $\theta=3^{\circ}$, which around $\theta=7^{\circ}$ changes into a single peak at the $L_{3}$ edge and disappears at the $L_{3}$ edge. Also, the asymmetry ratios show a wealth of detailed structure, and the spectra in Fig. 10

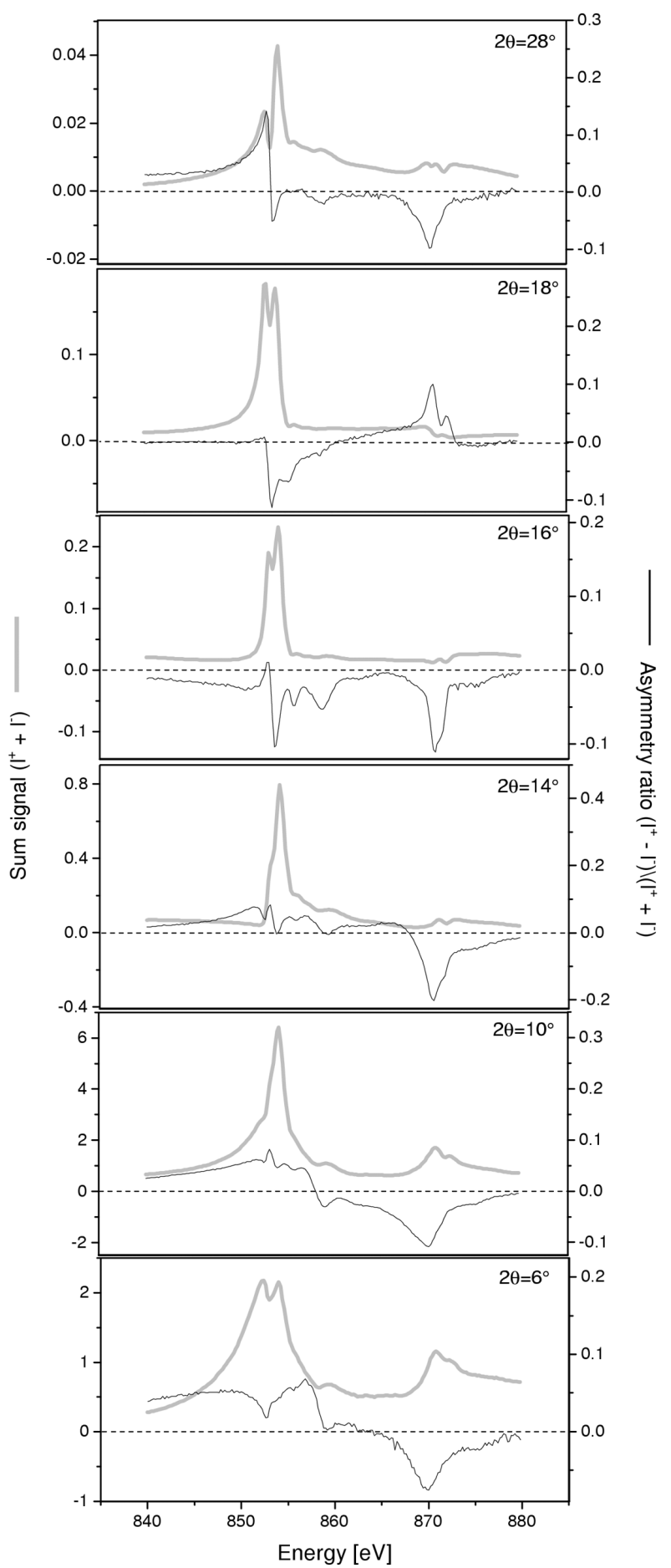

Figure 10

Photon energy dependence across the Ni $L_{2,3}$ resonance for the sum signal $\left(I^{+}+I^{-}\right)$(thick grey line) and the asymmetry ratio $\left(I^{+}-I^{-}\right) /\left(I^{+}+I^{-}\right)$(thin black line) of the specular reflectivity measured for different grazing-incidence angles in geometry $B$. The signal $I^{+}\left(I^{-}\right)$is defined as the specular reflectivity obtained by aligning the magnetization parallel (antiparallel) to the helicity vector of the X-rays. are distinctly different from the reflectivity results on a $\mathrm{Ni}(110)$ crystal reported by Sacchi \& Mirone (1998). The dichroic spectra in Fig. 10 can be regarded as an extension of X-ray magnetic circular dichroism (XMCD) in absorption (i.e. forward scattering). Compared with the absorption signal in XMCD, the scattering signal also includes the dispersive part of the scattering factor. The size of the dichroism in reflectivity can exceed that observed in XMCD. However, the energy dependence of the reflectivity (Fig. 10) is more complicated than that of the XMCD. While the integrated signals in XMCD can be related to the orbital and spin magnetic moments (Thole et al., 1992), this is much more difficult in the case of scattering. In order to apply the sum rules, the integral of the imaginary part of magnetic scattering factor would have to be extracted. However, the sum rules do not apply in the case of interference scattering where the charge scattering resonance varies with energy.

\section{Summary}

We have performed SXRMS measurements on a magnetically modulated, but chemically homogenous, Ni layer in a $\mathrm{Cu} / \mathrm{Ni} / \mathrm{Cu}$ system. Although this is both structurally and magnetically a rather complicated system for scattering studies, the preliminary data analysis looks very promising. Specular rod scans were used to study the structural profile of the sample. The oscillations owing to Kiessig fringes observed in the specular rod scans with different photon energies in the vicinity of the element-specific absorption edges allowed us to determine the corresponding layer thicknesses of the sample. However, at low angles an extra dip in the reflectivity is observed that does not appear in the case of multilayers with in-plane homogeneity. We ascribe this extra feature to interference from the in-plane periodicity in the sample.

Rocking scans were used to study the in-plane periodicity of the sample. Diffraction peaks are observed with a spacing of $0.0001 \AA^{-1}$, corresponding to a periodicity of the wires of $6 \mu \mathrm{m}$. Scans taken near the critical angle, where the X-rays penetrate only the capping layer, are still revealing a periodicity. This suggests that also the $\mathrm{Cu}$ capping has an in-plane periodic structure, possibly owing to a different oxidation of the single-crystalline and polycrystalline regions. At higher Bragg angles we observe an array of diffraction peaks, clearly showing that the sample diffracts like a grating. At the resonance of the $\mathrm{Ni} L_{2,3}$ and $\mathrm{Cu} L_{2,3}$ edges the specular peak exhibits extra structure and all diffraction peaks have an additional shoulder on the left-hand side. We ascribe this to interference of the grating profile at different depths in the sample.

We have demonstrated the coexistence of in-plane and perpendicularly magnetized domains in the sample from the selectivity for the magnetization direction using $\sigma$-polarized and circularly polarized light. First, there is a difference in the $\mathrm{Ni} L_{2,3}$-edge specular reflection after aligning the magnetization of all domains in-plane using an applied magnetic field. Second, the MCD in the reflectivity is different for the coexisting magnetic domain configuration and the inplane magnetic configuration.

The magnetic depth profile was measured by the MCD in reflectivity. At the $\mathrm{Ni} L_{3}$ resonance energy the Kiessig fringes in the specular rod scan are strongly suppressed compared with those at the $\mathrm{Ni} L_{2}$ energy owing to the reduced X-ray penetration length. The asymmetry ratio in reflectivity across the $\mathrm{Ni} L_{2,3}$ edge is very strong, showing a wealth of detailed structure. The spectral changes as a function of Bragg angle are remarkably strong.

A more quantitative analysis would be required to explore in detail the in-plain chemical and magnetic arrangement as well as the 
interdomain roughness. Calculations are in progress and will be presented in the near future.

We thank K. Larsson, P. Bencok and N. B. Brookes for their expert experimental assistance on the beamline.

\section{References}

Alders, D., Hibma, T., Sawatzky, G. A., Cheung, K. C., van Dorssen, G. E., Padmore, H. A., Roper, M. D., van der Laan, G., Vogel, J. \& Sacchi, M. (1997). J. Appl. Phys. 82, 3120-3124.

Als-Nielsen, J. \& McMorrow, D. (2000). Elements of Modern X-ray Physics. New York: Wiley.

Bland, J. A. C., Lew, W. S., Li, S. P., Lopez-Diaz, L., Vaz, C. A. F., Natali, M. \& Chen, Y. (2002). J. Phys. D, 35, 2384-2390.

Chesnel, K., Belakhovsky, M., Landis, S., Rodmacq, B., Dudzik, E., Collins, S. P., Dhesi, S. S. \& van der Laan, G. (2001). IEEE Trans. Magn. 37, 16611663.

Chesnel, K., Belakhovsky, M., Landis, S., Toussaint, J. C., Collins, S. P., van der Laan, G., Dudzik, E. \& Dhesi, S. S. (2002). Phys. Rev. B, 66, 024435/1-9.

Cowburn, R. P., Adeyeye, A. O. \& Bland, J. A. C. (1997). Appl. Phys. Lett. 70, 2309-2311.

Daillant, J. \& Gibaud, A. (1999). X-ray and Neutron Reflectivity: Principles and Applications, Lecture Notes in Physics 58. Berlin: Springer.

Dhesi, S. S., Mirone, A., De Nadai, C., Ohresser, P., Bencok, P., Brookes, N. B., Reutler, P., Revcolevschi, A., Tagliaferri, A., Toulemonde, O. \& van der Laan, G. (2004). Phys. Rev. Lett. 92, 056403/1-4.

Dudzik, E., Dhesi, S. S., Dürr, H. A., Collins, S. P., Roper, M. D., van der Laan, G., Chesnel, K., Belakhovsky, M., Marty, A. \& Samson, Y. (2000). Phys. Rev. B, 62, 5779-5785.

Dürr, H. A., Dudzik, E., Dhesi, S. S., Goedkoop, J. B., van der Laan, G., Belakhovsky, M., Mocuta, C., Marty, A. \& Samson, Y. (1999). Science, 284, 2166-2168.
Ebels, U., Radulescu, A., Henry, Y., Piraux, L. \& Ounadjela, K. (2000). Phys. Rev. Lett. 84, 983-986.

Hannon, J. P., Trammell, G. T., Blume, M. \& Gibbs, D. (1988). Phys. Rev. Lett. 61, 1245-1248.

Jaouen, N., Tonnerre, J. M., Raoux, D., Bontempi, E., Ortega, L., Muenzenberg, M., Felsch, W., Rogalev, A., Dürr, H. A., Dudzik, E., van der Laan, G., Suzuki, M. \& Maruyama, H. (2002). Phys. Rev. B, 66, 134420/ $1-14$.

Kao, C.-C., Chen, C. T., Johnson, E. D., Hastings, J. B., Lin, H. J., Ho, G. H., Meigs, G., Brot, J.-M., Hulbert, S. L., Idzerda, Y. U. \& Vettier, C. (1994). Phys. Rev. B, 50, 9599-9602.

Krusin-Elbaum, L., Shibauchi, T., Argyle, B., Gignac, L. \& Weller, D. (2001). Nature (London), 410, 444-446.

Laan, G. van der (1999). J. Magn. Magn. Mater. 192, 297-304.

Laan, G. van der, Dudzik, E., Collins, S. P., Dhesi, S. S., Dürr, H. A., Belakhovsky, M., Chesnel, K., Marty, A., Samson, Y. \& Gilles, B. (2000). Physica B, 283, 171-174.

Laan, G. van der, Dürr, H. A., Dudzik, E., Roper, M. D., Collins, S. P., Hase, T. P. A. \& Pape, I. (1999). Synchrotron Rad. News, 12(3), 5-9.

Laan, G. van der \& Thole, B. T. (1991). Phys. Rev. B, 43, 13401-13411.

Li, S. P., Lew, W. S., Bland, J. A. C., Lopez-Diaz, L., Vaz, C. A. F., Natali, M. \& Chen, Y. (2002). Phys. Rev. Lett. 88, 087202/1-4.

Lovesey, S. W. \& Collins, S. P. (1996). X-ray Scattering and Absorption by Magnetic Materials. Oxford University Press.

O’Brien, W. L. \& Tonner, B. P. (1994). Phys. Rev. B, 49, 15370-15373.

Sacchi, M. \& Mirone, A. (1998). Phys. Rev. B, 57, 8408-8415.

Sève, L., Jaouen, N., Tonnerre, J. M., Raoux, D., Bartolomé, F., Arend, M., Felsch, W., Rogalev, A., Goulon, J., Gautier, C. \& Bérar, J. F. (1999). Phys. Rev. B, 60, 9662-9674.

Taniyama, T., Nakatani, I., Namikawa, T. \& Yamazaki, Y. (1999). Phys. Rev. Lett. 82, 2780-2783.

Thole, B. T., Carra, P., Sette, F. \& van der Laan, G. (1992). Phys. Rev. Lett. 68 , 1943-1946.

Tonnerre, J. M., Sève, L, Barbara-Dechelette, A., Bartolomé, F., Raoux, D. Chakarian, V., Kao, C. C., Fischer, H., Andrieu, S. \& Fruchart, O. (1998). J. Appl. Phys. 83, 6293-6295. 